\title{
Brittle Superconducting Magnets: An Equivalent Strain Model
}

\author{
E. Barzi, M. Danuso
}

\begin{abstract}
To exceed fields of $10 \mathrm{~T}$ in accelerator magnets, brittle superconductors like $\mathrm{A15} \mathrm{Nb}_{3} \mathrm{Sn}$ and $\mathrm{Nb}_{3} \mathrm{Al}$ or ceramic High Temperature Superconductors have to be used. For such brittle superconductors it is not their maximum tensile yield stress that limits their structural resistance as much as strain values that provoke deformations in their delicate lattice, which in turn affect their superconducting properties. Work on the sensitivity of $\mathrm{Nb}_{3} \mathrm{Sn}$ cables to strain has been conducted in a number of stress states, including uniaxial [1-5] and multi-axial [6-8], producing usually different results. This has made the need of a constituent design criterion imperative for magnet builders. In conventional structural problems an equivalent stress model is typically used to verify mechanical soundness. In the superconducting community a simple scalar equivalent strain to be used in place of an equivalent stress would be an extremely useful tool. As is well known in fundamental mechanics, there is not one single way to reduce a multiaxial strain state as represented by a $2^{\text {nd }}$ order tensor to a scalar. The conceptual experiment proposed here will help determine the best scalar representation to use in the identification of an equivalent strain model.
\end{abstract}

Index Terms - Equivalent strain, uni-axial stress state, multiaxial stress state, critical current

\section{INTRODUCTION}

$\mathrm{W}$ ORK in literature on the sensitivity of $\mathrm{Nb}_{3} \mathrm{Sn}$ cables to strain has been conducted in a number of stress states, including uniaxial [1-5] and multi-axial [6-8] stress states, using some very different experimental setups. In order to determine a model that assigns to each tensor a scalar representation, we need data exploring all the dimensions of the tensor by means of an experiment that provides consistent and controlled conditions. For instance, for a 2D problem, we would need two load cases that produce a linearly independent strain tensor. As pointed out, the strain tensor should reach closer to the physics of the phenomenon than the stress tensor, since it is lattice deformations that affect the superconductor's properties.

\section{Equivalent STRAin MOdels}

Transport properties measurements in [1-8] were based on the principle of applying transverse pressure on a sample and

Manuscript received August 3, 2010.

This work was supported by the U.S. Department of Energy.

E. Barzi is with the Fermi National Accelerator Laboratory (Fermilab), P.O. Box 500, Batavia, IL 60510 USA (phone: 630-840-3446; fax: 630-8403369; e-mail: barzi@fnal.gov). M. Danuso is with Finmeccanica. then measuring its critical current $\mathrm{I}_{\mathrm{c}}$. We can think of at least two different boundary conditions for the cable, which may produce different current density performances: plane stress with free sides, and plane stress with constrained sides (Fig. $1)$.

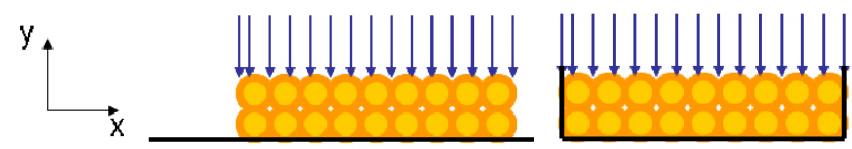

Fig. 1. Uni-axial case A, free sides (left), and multi-axial case B (right).

The first load case, uni-axial case A, represented in Fig. 1 (left), has $\sigma_{y y}=-p$ and $\sigma_{x x}=\sigma_{z z}=0$. It can therefore be represented by the matrix:

$$
\left(\begin{array}{ccc}
0 & 0 & 0 \\
0 & -p & 0 \\
0 & 0 & 0
\end{array}\right)
$$

The strain is given by:

$$
\left(\begin{array}{ccc}
v \frac{p}{E} & 0 & 0 \\
0 & -\frac{p}{E} & 0 \\
0 & 0 & v \frac{p}{E}
\end{array}\right)
$$

where $v$ is the Poisson coefficient and $\mathrm{E}$ the Young modulus.

The second load case, multi-axial case $\mathrm{B}$, represented in Fig. 1 (right), has $\sigma_{y y}=-p, \sigma_{x x}=-v p$ and $\sigma_{z z}=0$. To generalize this load case, we suppose to give a preload on the $\mathrm{x}$ direction equal to $\varepsilon_{\mathrm{xx} 0}$. The strain tensor can then be represented by:

$$
\left(\begin{array}{ccc}
\varepsilon_{x x 0} & 0 & 0 \\
0 & \left(v^{2}-1\right) \frac{p}{E}-v \varepsilon_{x x 0} & 0 \\
0 & 0 & v(1+v) \frac{p}{E}-v \varepsilon_{x x 0}
\end{array}\right)
$$

The following step defines a list of three possible equivalent strain models. For each equivalent strain model, a prediction of the performance of load case B with respect to load case A can be made. This prediction can then be matched to the experiment outcome to determine which model best describes the real phenomenon. 
A. Maximum Absolute Eigenvalue, or $\max \left|\varepsilon_{i}\right|$,

which is $\mathrm{p} / \mathrm{E}$ in case $\mathrm{A}$, and

$$
\max \left[\left|\left(1-v^{2}\right) \frac{p}{E}+v \varepsilon_{x x 0}\right|,\left|v(1+v) \frac{p}{E}-v \varepsilon_{x x 0}\right|,\left|\varepsilon_{x x 0}\right|\right]
$$

in case $\mathrm{B}$, therefore the candidate equivalent strains are:

$$
\begin{aligned}
& \varepsilon_{e q A}=\frac{p}{E} \\
& \varepsilon_{e q B}=\max \left[\left|\left(1-v^{2}\right) \frac{p}{E}+v \varepsilon_{x x 0}\right|,\left|v(1+v) \frac{p}{E}-v \varepsilon_{x x 0}\right|,\left|\varepsilon_{x x 0}\right|\right]
\end{aligned}
$$

\section{B. Maximum Angular Deformation,}

i.e. the maximum diameter of the Mohr's circles of strain, or $\max \left|\varepsilon_{\mathrm{i}}-\varepsilon_{\mathrm{j}}\right|$, gives the following candidate equivalent strains:

$$
\begin{aligned}
& \varepsilon_{e q A}=(1+v) \frac{p}{E} \\
& \varepsilon_{e q B}=\max \left[(1+v) \frac{p}{E},\left|v(1+v) \frac{p}{E}-(1+v) \varepsilon_{x x 0}\right|,\left|\left(1-v^{2}\right) \frac{p}{E}+(1+v) \varepsilon_{x x 0}\right|\right]
\end{aligned}
$$

\section{Maximum Angular Deformation on the Cross Sectional Plane,}

i.e. an equivalent strain proportional to the modulus of the difference between the principal stresses on the cross-sectional plane, or mod $\left|\varepsilon_{1}-\varepsilon_{2}\right|$, produces the following candidate equivalent strains:

$$
\begin{aligned}
& \varepsilon_{\text {eqA }}=(1+v) \frac{p}{E} \\
& \varepsilon_{\text {eqB }}=\left|\left(1-v^{2}\right) \frac{p}{E}+(1+v) \varepsilon_{x x 0}\right|
\end{aligned}
$$

\section{Models PREDictions}

\section{A. Maximum Eigenvalue}

In Fig. 2 the equivalent strains are shown for the Maximum Eigenvalue model as functions of the transverse pressure, for a fixed Poisson coefficient of 0.3 , and a Young's Modulus of 40 GPa. For load case $B, \varepsilon_{x x 0}=-0.001$. To predict the effect of constraining the sample on the $I_{c}$ of the superconducting cable according to each model, the $I_{c}$ data obtained from the uniaxial experiment can be used. The $I_{c}$ obtained in the uni-axial test for increasing transverse pressure values is correlated to the equivalent strain value indicated by each model. This is shown for instance in Fig. 3 for the Maximum Eigenvalue representation, where data from a Restack Rod Process $\mathrm{Nb}_{3} \mathrm{Sn}$ conductor were used. The correlation found for each model between $I_{c}$ and equivalent strain is then used to predict the $I_{c}$ in the case of the constrained sample (multi-axial stress state).
The prediction obtained for the Maximum Eigenvalue model is shown in Fig. 4 in the same graph as the uni-axial data. This model predicts a lower sensitivity to transverse pressure with respect to the uni-axial configuration.

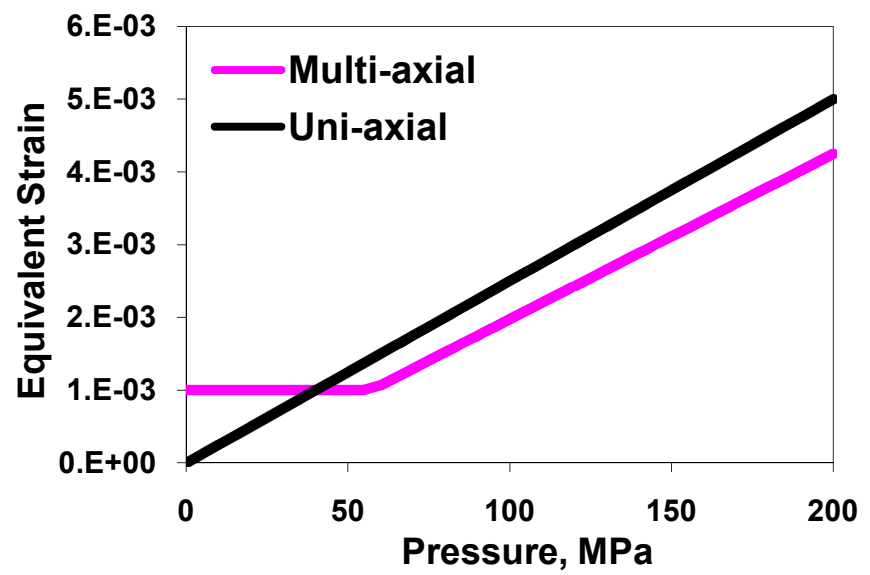

Fig. 2. Equivalent strains as a function of transverse pressure at fixed Poisson coefficient $(v=0.3)$ for cases $\mathrm{A}$ and $\mathrm{B}$ in the Maximum Eigenvalue representation.

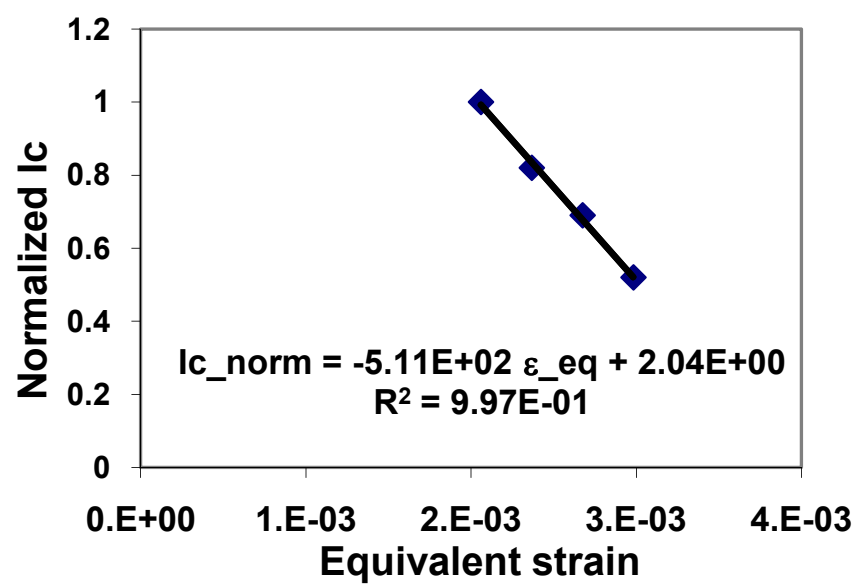

Fig. 3. Correlation between the critical current obtained in the uni-axial test for increasing transverse pressure values with the equivalent strain indicated in the Maximum Eigenvalue representation.

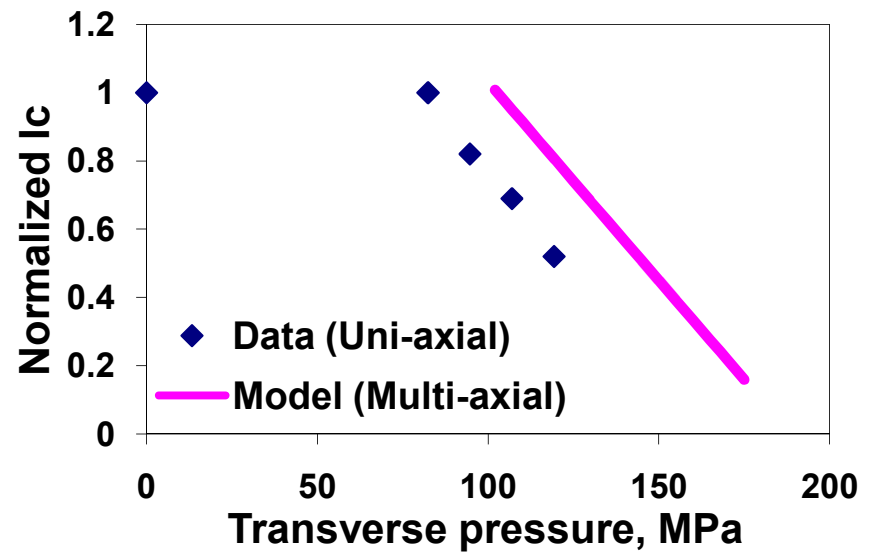

Fig. 4. Predictions of normalized critical current vs. transverse pressure for a constrained sample according to the Maximum Eigenvalue strain model.

\section{B. Maximum Angular Deformation}

In Fig. 5 the equivalent strains are reported for the 
Maximum Angular Deformation model as functions of the transverse pressure, for a fixed Poisson coefficient of 0.3 , and a Young's Modulus of $40 \mathrm{GPa}$. For load case B, $\varepsilon_{\mathrm{xx} 0}=-0.001$. The prediction obtained for the Maximum Angular Deformation model is shown in Fig. 6 in the same graph as the uni-axial data. This model predicts that one would find the same sensitivity to transverse pressure in the uni-axial and multi-axial states.

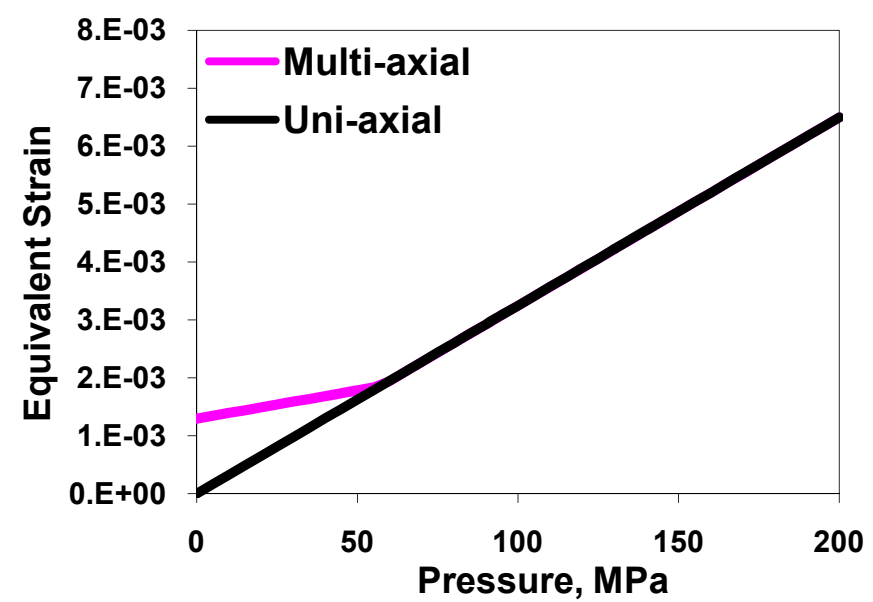

Fig. 5. Equivalent strains as a function of transverse pressure at fixed Poisson coefficient $(v=0.3)$ for cases A and B in the Maximum Angular Deformation representation.

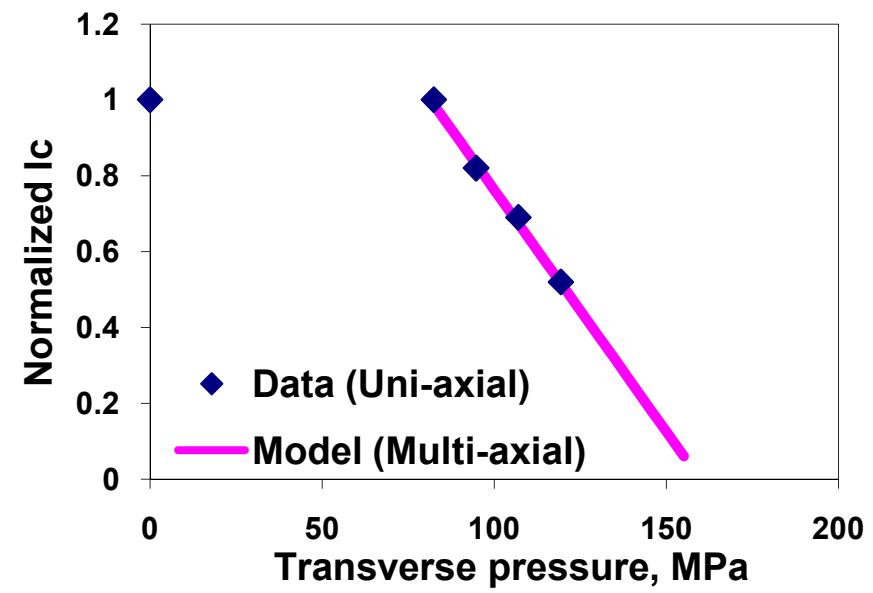

Fig. 6. Predictions of normalized critical current vs. transverse pressure for a constrained sample according to the Maximum Angular Deformation strain model.

\section{Maximum Angular Deformation on the Cross Section}

In Fig. 7 the equivalent strains are reported for the model of Maximum Angular Deformation on the cross section as functions of the transverse pressure, for a fixed Poisson coefficient of 0.3, and a Young's Modulus of $40 \mathrm{GPa}$. For load case $\mathrm{B}, \varepsilon_{\mathrm{xx} 0}=-0.001$. The prediction obtained for the model of Maximum Angular Deformation on the cross section is shown in Fig. 8 in the same graph as the uni-axial data. This model predicts the largest difference in transverse pressure sensitivity between the uni-axial and the multi-axial state.

The experiment herein proposed of testing the same sample in uni-axial and multi-axial configurations is presently just a conceptual experiment. Using data in literature can be attempted to check whether they allow determining the soundness of any equivalent strain model. Fig. 9 shows predictions of normalized critical current vs. transverse pressure for a constrained sample using the Maximum Eigenvalue model and the model of Maximum angular deformation on the cross section compared with uni-axial data on a Modified Jelly-Roll (MJR) cable [4] and the test of an older MJR cable [8].

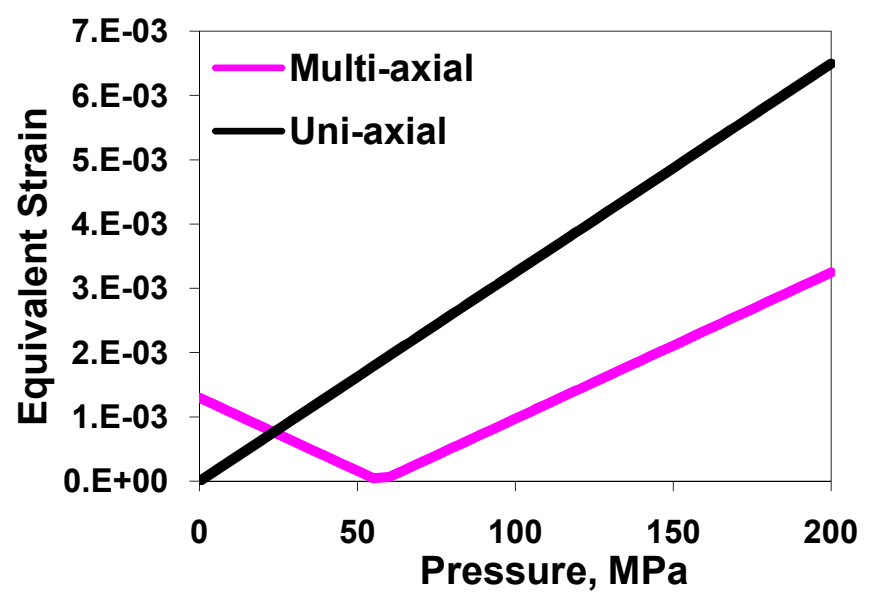

Fig. 7. Equivalent strains as a function of transverse pressure at fixed Poisson coefficient $(v=0.3)$ for cases A and B in the Maximum angular deformation on the cross section representation.

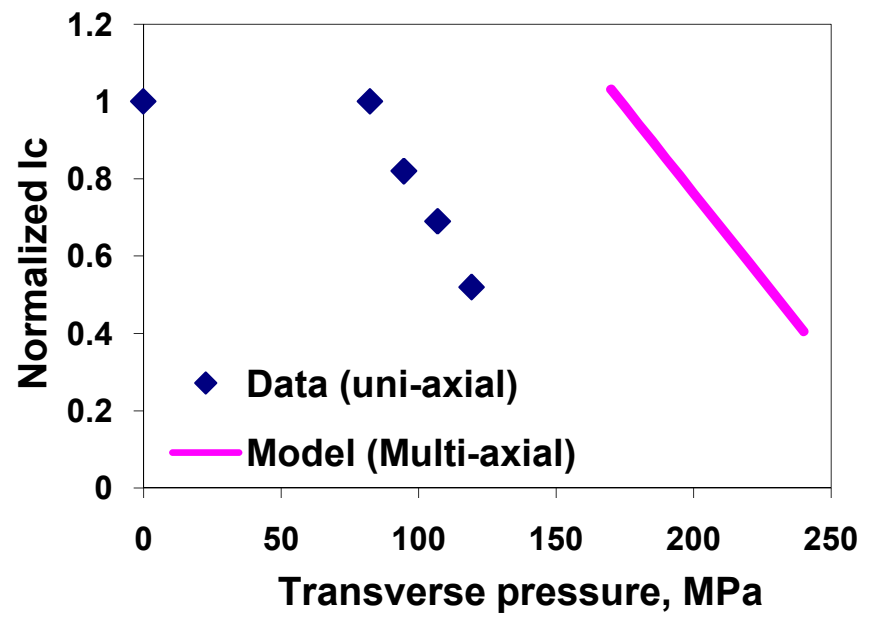

Fig. 8. Predictions of normalized critical current vs. transverse pressure for a constrained sample according to the Maximum angular deformation on the cross section strain model.

\section{CONCLUSIONS}

Whereas the Maximum Angular Deformation model predicts no difference in critical current performance between samples in uni-axial and multi-axial stress states at same transverse pressure, the Maximum angular deformation on the cross sectional plane is the model that predicts the largest difference. Existing data on even similar conductors tested in different setups do not allow discerning the best model. A controlled experiment to represent multi-axial load case B would point to whether one of these or other equivalent strain models represented correctly the electrical performance of brittle superconducting materials. If so, the successful equivalent strain model could be used as a constituent criterion 
in magnet design. In addition, by representing cable transport properties as a function of such equivalent strain rather than as a function of transverse pressure, the various experimental setups where samples are subjected to different stress states should provide more consistent results.

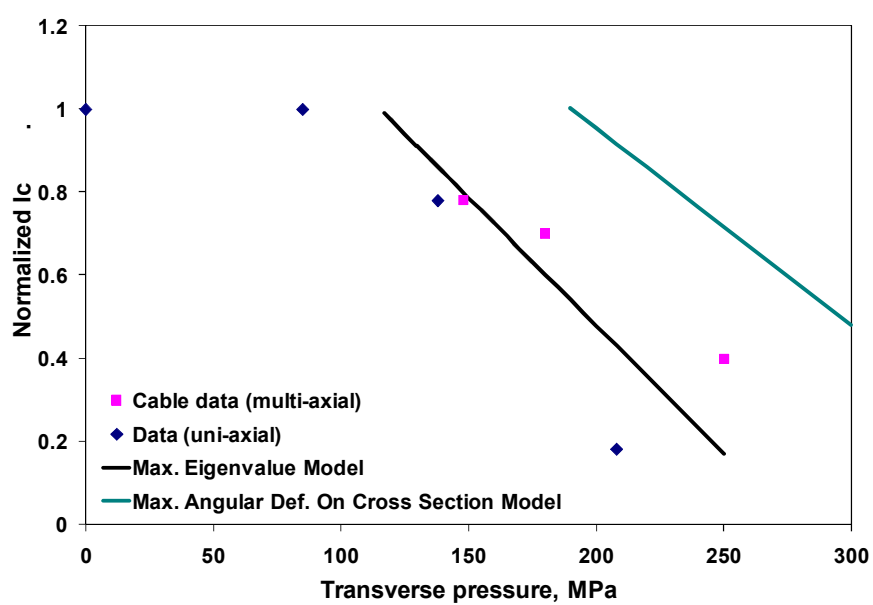

Fig. 9. Predictions of normalized critical current vs. transverse pressure for a constrained sample using the Maximum Eigenvalue model and the model of Maximum angular deformation on the cross section compared with uni-axial data on a MJR cable [4] and a MJR cable test [8].

\section{ACKNOWLEDGMENT}

The authors wish to thank Prof. Marco Beghini who inspired this work.

\section{REFERENCES}

[1] "A Device to Test Critical Current Sensitivity of $\mathrm{Nb}_{3}$ Sn Cables to Pressure", E. Barzi, M. Fratini, A. V. Zlobin. Advances in Cryogenic Engineering, V. 48, p. 45 (2002).

[2] "Sensitivity of $\mathrm{Nb}_{3}$ Sn Rutherford-type Cables to Transverse Pressure", E. Barzi, T. Wokas, A. V. Zlobin. 6th European Conference on Applied Superconductivity (EUCAS 2003), Sept. 14-18, 2003, Sorrento (Naples), Italy.

[3] "Sensitivity of $\mathrm{Nb}_{3}$ Sn Rutherford-type Cables to Transverse Pressure", E. Barzi, T. Wokas, A. V. Zlobin. IEEE Trans. Appl. Sup., V. 15, No. 2, p. 1541 (2005).

[4] "Effect of Transverse Pressure on Brittle Superconductors", E. Barzi et al.. IEEE Trans. Appl. Sup., V. 18, No. 2, p. 980 (2008).

[5] " $\mathrm{Cu}$ Stabilized $\mathrm{Nb}_{3} \mathrm{Al}$ Strands for the High Field Accelerator Magnet", A. Kikuchi et al.. IEEE Trans. Appl. Sup., V. 18, No. 2, p. 1026 (2008).

[6] "The Reduction of the Critical Current in $\mathrm{Nb}_{3} \mathrm{Sn}$ Cables under Transverse Loads", J. M. van Oort et al.. IEEE Trans. Appl. Sup., V. 3, No. 1, p. 559 (1993).

[7] "The Effect of Transverse Load up to $300 \mathrm{MPa}$ on the Critical Current of $\mathrm{Nb}_{3} \mathrm{Sn}$ Cables", H. Boschman et al., IEEE Trans. Magn., V. 27, No. 2, p. 1829 (1991).

[8] "Critical Current of Superconducting Rutherford Cable in High Magnetic Fields with Transverse Pressure", D. R. Dietderich et al.. IEEE Trans. Appl. Sup., V. 9, No. 2, p. 122 (1999). 